\title{
Design for Motor Controller in Hybrid Electric Vehicle Based on Vector Frequency Conversion Technology
}

\author{
Jing Lian, Yafu Zhou, Teng Ma, and Wei Wang \\ School of Automotive Engineering, Faculty of Vehicle Engineering and Mechanics, State Key Laboratory of \\ Structural Analysis for Industrial Equipment, Dalian University of Technology, Dalian 116024, China \\ Correspondence should be addressed to Yafu Zhou, dlzyf62@126.com
}

Received 26 July 2009; Accepted 30 September 2009

Academic Editor: José Balthazar

Copyright (c) 2010 Jing Lian et al. This is an open access article distributed under the Creative Commons Attribution License, which permits unrestricted use, distribution, and reproduction in any medium, provided the original work is properly cited.

\begin{abstract}
Motor and its control technology are one of the main components of Hybrid Electric Vehicle (HEV). To meet HEV's fast torque response, vector control algorithm based on rotor fluxoriented and simulation model is concerned and modular designs for controller's hardware and software are presented in the paper in order to build a platform to achieve the vector control of asynchronous induction motor. Analyze the controller's electromagnetic compatibility, introduce the corresponding antijamming measures to assure the normal operation of the electromagnetic sensitive devices such as CAN bus; experiment proves that the measure is practical and feasible. On the basis of the control logic correct, such as improving CAN bus communication reliability, assuring power-on sequence and fault treatment, carry on the motor bench experiment, test its static properties, and adjust the controller parameters. The experimental results show that the designed driving system has the performance of low speed and high torque, a wide range of variable speed and high comprehensive efficiency.
\end{abstract}

\section{Introduction}

Hybrid Electric Vehicle (HEV) is the vehicle with two or more power supplies (one of these is electrical energy) $[1,2]$, such as unit of internal combustion engine and secondary cell or unit of fuel cell and secondary cell. At present, developing HEV research is one of the most meaningful ways to solve the problems of pollution and energy.

Drive motor is one of the HEV's core components. In accordance with different vehicles' operating environment of level road, ramp, acceleration-deceleration, start-stop, and so on, the motor operating mode switches frequently among fractional load, heavy load, and excess load, hence improving the integrated efficiency of the electrical motor is a key 
issue for the development of motor and its controller, which directly affects vehicle's power performance, fuel economy, and emission. The basic performance requirements of HEV's motor drive system $[3,4]$ are high-performance and low-loss, high power density, low speed and high torque, a wide range of variable speed, strong overload capacity, good reliability, and so on. Based on the analysis of HEV's mainstream motor drive systems in the present market, this paper chooses the AC asynchronous induction motor which has low cost and high efficiency as a drive motor selection.

The AC asynchronous induction motor is the nonlinear time-varying system [5] of high-order, multivariable, and strong coupling, hence the control method based on motor's static mathematical model has been unable to meet the requirements of its dynamic performance. Currently, the control methods of AC asynchronous induction motor in HEV are mainly two kinds of direct torque control and vector control [6]. In the low speed, direct torque control is easy to produce torque fluctuation and no closed-loop current and is easy to produce over-current, which cannot meet the requirements of high stability torque when the vehicle is in the low-speed climbing state, at the same time there are the problems of motor temperature rise and high noise. Vector control method is able to achieve the AC asynchronous induction motor's decoupling control of magnetic flux and torque, which has good torque control characteristics, being analogous to DC motor, and can improve the efficiency of drive system and achieve maximum efficiency control. In addition, vector control can achieve a fixed switching frequency PWM modulation and reduce the harmonic content of the motor current, which, to a certain extent, reduce the motor temperature rise and noise.

Therefore, this paper introduces the vector control algorithm based on the rotor flux linkage oriented and conducts research to design the motor controller of Hybrid Electric Vehicle with high performance, high efficiency, and high reliability. First, introduce the basic principles of algorithm, build the modularized simulation platform, and then design the controller's software and hardware; meanwhile, analyze controller's electromagnetic compatibility and take appropriate antijamming measures to ensure the normal operation of the electromagnetic sensitive equipments such as CAN bus. Finally, carry out the motor bench experiment on the basis of the control logic correct, such as improving the CAN bus communication reliability, ensuring the power-on sequence, and fault treatment, and complete the performance experiment of the motor drive system.

\section{Vector Control Based on Rotor Flux Linkage Oriented}

In terms of $\mathrm{AC}$ asynchronous motor, the current, voltage, magnetic flux and electromagnetic torque are interrelated. The basic idea of vector control $[7,8]$ uses the mathematical coordinate transformation method to transform current $i_{A}, i_{B}, i_{C}$ of AC three-phrase winding $A, B, C$ to $i_{\alpha}, i_{\beta}$ of two-phrase static winding $\alpha, \beta$, once again by mathematical coordinate transformation transform $i_{\alpha}, i_{\beta}$ to direct current $i_{M}, i_{T}$ of two-phrase rotating winding $M, T$. In essence, through mathematical coordinate transformation transform stator current of AC asynchronous motor into two components, one is excitation component $i_{M}$ which is used to generate rotating magnetic potential, and another is torque component $i_{T}$ which is used to generate electromagnetic torque, as shown in Figure 1. Adjusting $i_{M}$ can adjust the strength of the magnetic field, and adjusting $i_{T}$ can adjust the size of torque in the constant magnetic field. 


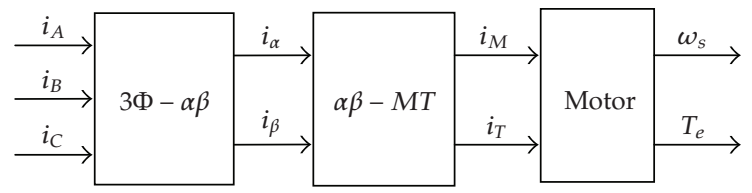

Figure 1: Basic idea of vector control in asynchronous motor.

\subsection{Coordinate Transformation}

(1) Transform static three-phrase coordinate system $A, B, C$ into static two-phrase coordinate system $\alpha-\beta$.

Take $\alpha$ axis to be coincident with $A$ axis, the effective turn of each phrase in the three-phrase winding is $N_{3}$, the effective turn of each phrase in the two-phrase winding is $N_{2}, N_{3} / N_{2}=$ $\sqrt{2 / 3}$. In order to facilitate the inverse transform, add an imaginary zero axis current $i_{0}$, and $i_{0}=K\left(i_{A}+i_{B}+i_{C}\right)=0, K$ is an undetermined constant. Take the principle that the total magnetic potential and total power are permanent before and after transformation, there is

$$
\left[\begin{array}{l}
i_{\alpha} \\
i_{\beta} \\
i_{0}
\end{array}\right]=\sqrt{\frac{2}{3}}\left[\begin{array}{ccc}
1 & -\frac{1}{2} & -\frac{1}{2} \\
0 & \sqrt{\frac{3}{4}} & -\sqrt{\frac{3}{4}} \\
\sqrt{\frac{1}{2}} & \sqrt{\frac{1}{2}} & \sqrt{\frac{1}{2}}
\end{array}\right]\left[\begin{array}{l}
i_{A} \\
i_{B} \\
i_{C}
\end{array}\right] .
$$

(2) Transform rotating two-phrase coordinate system into static two-phrase coordinate system.

Rotating two-phrase coordinate system are represented by $d$ axis and $q$ axis which are perpendicular to each other, the angle between $d$ axis and $\alpha$ axis is $\theta$, the rotating speed of $d-q$ axis is $\omega, \omega=d \theta / d t$, then there is

$$
\left[\begin{array}{l}
i_{\alpha} \\
i_{\beta}
\end{array}\right]=\left[\begin{array}{cc}
\cos \theta & -\sin \theta \\
\sin \theta & \cos \theta
\end{array}\right]\left[\begin{array}{l}
i_{d} \\
i_{q}
\end{array}\right] .
$$

\subsection{The Motor Equations in Different Coordinate System}

Define stator current $i_{A}, i_{B}, i_{C}$, rotor current $i_{a}, i_{b}, i_{c}$, stator flux linkage $\Psi_{A}, \Psi_{B}, \Psi_{C}$, rotor flux linkage $\Psi_{a}, \Psi_{b}, \Psi_{c}$. The motor equations in different coordinate system are shown as follows 


\section{(1) Three-Phrase Static Coordinate System}

We have the equation of flux linkage

$$
\left[\begin{array}{l}
\Psi_{A} \\
\Psi_{B} \\
\Psi_{C} \\
\Psi_{a} \\
\Psi_{b} \\
\Psi_{c}
\end{array}\right]=\left[\begin{array}{llllll}
L_{A A} & L_{A B} & L_{A C} & L_{A a} & L_{A b} & L_{A c} \\
L_{B A} & L_{B B} & L_{B C} & L_{B a} & L_{B b} & L_{B c} \\
L_{C A} & L_{C B} & L_{C C} & L_{C a} & L_{C b} & L_{C c} \\
L_{a A} & L_{a B} & L_{a C} & L_{a a} & L_{a b} & L_{a c} \\
L_{b A} & L_{b B} & L_{b C} & L_{b a} & L_{b b} & L_{b c} \\
L_{c A} & L_{c B} & L_{c C} & L_{c a} & L_{c b} & L_{c c}
\end{array}\right]\left[\begin{array}{c}
i_{A} \\
i_{B} \\
i_{C} \\
i_{a} \\
i_{b} \\
i_{c}
\end{array}\right],
$$

where $L_{A A}, L_{B B}, L_{C C}$, respectively, denote the self-inductance of stator's each phrase and $L_{A A}=L_{B B}=L_{C C}=L_{m 1}+L_{e 1}$, and where $L_{a a}, L_{b b}, L_{c c}$, respectively, denote the self-inductance of rotor's each phrase and $L_{a a}=L_{b b}=L_{c c}=L_{m 2}+L_{e 2}$. Because the stator turns are equivalent to rotor turns after conversion, $L_{m 1}=L_{m 2}$. We have mutual inductance:

$$
\begin{gathered}
L_{A B}=L_{B A}=\cdots=L_{C B}=L_{m 1} \cos 120^{\circ}=-0.5 L_{m 1}, \\
L_{a b}=L_{b a}=\cdots=L_{c b}=L_{m 1} \cos 120^{\circ}=-0.5 L_{m 1}, \\
L_{A a}=L_{a A}=\cdots=L_{c C}=L_{m 1} \cos \theta \\
L_{A b}=L_{b A}=\cdots=L_{a C}=L_{m 1} \cos \left(\theta+120^{\circ}\right), \\
L_{A c}=L_{c A}=\cdots=L_{b C}=L_{m 1} \cos \left(\theta-120^{\circ}\right),
\end{gathered}
$$

where $L_{e 1}$ denotes stator's leakage inductance and $L_{e 2}$ denotes rotor's leakage inductance $L_{m 1}$ denotes mutual inductance between each stator and $L_{m 2}$ denotes mutual inductance between each rotor.

There is equation of voltage

$$
\left[\begin{array}{l}
u_{A} \\
u_{B} \\
u_{C} \\
u_{a} \\
u_{b} \\
u_{c}
\end{array}\right]=\left[\begin{array}{cccccc}
R_{1} & 0 & 0 & 0 & 0 & 0 \\
0 & R_{1} & 0 & 0 & 0 & 0 \\
0 & 0 & R_{1} & 0 & 0 & 0 \\
0 & 0 & 0 & R_{2} & 0 & 0 \\
0 & 0 & 0 & 0 & R_{2} & 0 \\
0 & 0 & 0 & 0 & 0 & R_{2}
\end{array}\right]\left[\begin{array}{c}
i_{A} \\
i_{B} \\
i_{C} \\
i_{a} \\
i_{b} \\
i_{c}
\end{array}\right]+p\left[\begin{array}{c}
\Psi_{A} \\
\Psi_{B} \\
\Psi_{C} \\
\Psi_{a} \\
\Psi_{b} \\
\Psi_{c}
\end{array}\right],
$$

where $u$ denotes voltage, $p$ denotes $d / d t, R_{1}$ and $R_{2}$, respectively, denote the resistance of stator winding and rotor winding. 
There is equation of torque. On the condition that the current is constant and only mechanical displacement changes, electromagnetic torque is equivalent to the partial derivative of the magnetic field energy to mechanical angular displacement.

$$
\begin{aligned}
T_{e}= & \frac{1}{2} n_{p} i^{T} \frac{\partial L}{\partial \theta} i=\frac{1}{2} n_{p}\left[i_{s}^{T} \frac{\partial L_{r s}}{\partial \theta} i_{s}+i_{s}^{T} \frac{\partial L_{s r}}{\partial \theta} i_{r}\right] \\
=-n_{p} L_{m} & {\left[\left(i_{A} i_{a}+i_{B} i_{b}+i_{C} i_{c}\right) \sin \theta+\left(i_{A} i_{b}+i_{B} i_{c}+i_{C} i_{a}\right) \sin \left(\theta+120^{\circ}\right)\right.} \\
& \left.+\left(i_{A} i_{c}+i_{B} i_{a}+i_{C} i_{b}\right) \sin \left(\theta-120^{\circ}\right)\right]
\end{aligned}
$$

where $T_{e}$ denotes electromagnetic torque, $\theta$ denotes rotor space angular displacement which is shown by electrical angle, $n_{p}$ denotes the number of pole-pairs, and $L$ denotes inductance matrix of formula (2.3).

Equation of motion is

$$
T_{e}-T_{L}=\frac{J}{n_{p}} \frac{d \omega}{\partial t}
$$

where $T_{L}$ denotes load torque and $J$ denotes moment of inertia of units.

\section{(2) $d-q$ Rotating Coordinate System}

Given the mathematical model of the two-phrase motor, stator winding are $d_{1}$ and $q_{1}$ which is perpendicular to each other, and rotor winding is $d_{2}$ and $q_{2}$ which are also perpendicular to each other. In order to calculate conveniently, let $d_{1}$ and $q_{1}$ be, respectively, coincident with $d_{2}$ and $q_{2}$ which are, respectively, known as $d$ and $q$ axes. Let $\omega_{1}$ be the synchronous angular velocity of the stator frequency, $\omega_{11}$ rotational speed of $d$ axis relative to $A$ axis, and $\omega_{12}$ rotational speed of $d$ axis relative to $a$ axis. The equation of flux linkage is

$$
\left[\begin{array}{l}
\Psi_{d 1} \\
\Psi_{q 1} \\
\Psi_{d 2} \\
\Psi_{q 2}
\end{array}\right]=\left[\begin{array}{cccc}
L_{s} & 0 & L_{m} & 0 \\
0 & L_{s} & 0 & L_{m} \\
L_{m} & 0 & L_{r} & 0 \\
0 & L_{m} & 0 & L_{r}
\end{array}\right]\left[\begin{array}{c}
i_{d 1} \\
i_{q 1} \\
i_{d 2} \\
i_{q 2}
\end{array}\right]
$$

where $L_{m}$ denotes mutual inductance of stator winding and rotor winding and $L_{m}=$ $(3 / 2) L_{m 1}, L_{s}$ denotes self-inductance of stator winding and $L_{s}=L_{e 1}+(3 / 2) L_{m 1}$, and $L_{r}$ denotes self-inductance of rotor winding and $L_{r}=L_{e 2}+(3 / 2) L_{m 1}$.

Equation of voltage is

$$
\left[\begin{array}{l}
u_{d 1} \\
u_{q 1} \\
u_{d 2} \\
u_{q 2}
\end{array}\right]=\left[\begin{array}{cccc}
R_{1}+L_{s} p & -\omega_{11} L_{s} & L_{m} p & -\omega_{11} L_{m} \\
\omega_{11} L_{s} & R_{1}+L_{s} p & \omega_{11} L_{m} & L_{m} p \\
L_{m} p & -\omega_{12} L_{m} & R_{2}+L_{r} p & -\omega_{12} L_{r} \\
\omega_{12} L_{m} & L_{m} p & \omega_{12} L_{r} & R_{2}+L_{r} p
\end{array}\right]\left[\begin{array}{c}
i_{d 1} \\
i_{q 1} \\
i_{d 2} \\
i_{q 2}
\end{array}\right] .
$$


Equation of torque is

$$
T_{e}=n_{p} L_{m}\left(i_{q 1} i_{d 2}-i_{d 1} i_{q 2}\right)
$$

Equation of motion is same with formula (2.7).

\section{(3) Equation of Asynchronous Motor in M-T Coordinate System}

In order to make the system decoupling, define that $d$ axis is coincident with the direction of rotor flux linkage vector $\Psi_{2}$ which is entitled $M$ axis. The axis being perpendicular to $\Psi_{2}$ is $q$ axis which is entitled $T$ axis. Assuming an equal number of turns of the winding, we can eliminate the number of turns in the magnetic potential and the magnetic potential $F$ can be marked as vector $i_{1}$, that is, stator current and stator magnetic potential $F_{1}$ are only same with the direction of magnetic potential $F$.

Transform $d, q$ in formula (2.8) into $m, t$. Due to $M$ axis on $\Psi_{2}$ axis, $\Psi_{m 2}=\Psi_{2}, \Psi_{t 2}=0$, the new equation of flux linkage is

$$
\left[\begin{array}{l}
\Psi_{m 1} \\
\Psi_{t 1} \\
\Psi_{m 2} \\
\Psi_{t 2}
\end{array}\right]=\left[\begin{array}{cccc}
L_{s} & 0 & L_{m} & 0 \\
0 & L_{s} & 0 & L_{m} \\
L_{m} & 0 & L_{r} & 0 \\
0 & 0 & 0 & 0
\end{array}\right]\left[\begin{array}{c}
i_{m 1} \\
i_{t 1} \\
i_{m 2} \\
i_{t 2}
\end{array}\right]
$$

Given the characteristics that the terminal voltage of the cage asynchronous motor is zero, formula (2.9) can be simplified

$$
\left[\begin{array}{c}
u_{m 1} \\
u_{t 1} \\
0 \\
0
\end{array}\right]=\left[\begin{array}{cccc}
R_{1}+L_{s} p & -\omega_{1} L_{s} & L_{m} p & -\omega_{1} L_{m} \\
\omega_{1} L_{s} & R_{1}+L_{s} p & \omega_{1} L_{m} & L_{m} p \\
L_{m} p & 0 & R_{2}+L_{r} p & 0 \\
\omega_{s} L_{m} & 0 & \omega_{s} L_{r} & R_{2}+L_{r} p
\end{array}\right]\left[\begin{array}{c}
i_{m 1} \\
i_{t 1} \\
i_{m 2} \\
i_{t 2}
\end{array}\right]
$$

The equation of torque is same with formula (2.10) and the equation of motion is same with formula (2.7).

\subsection{Vector Control Algorithm and Simulation}

By formula (2.11) and (2.12), there are

$$
\begin{aligned}
& i_{m 2}=\frac{-p\left(L_{m} i_{m 1}+L_{r} i_{m 2}\right)}{R_{2}}=-\frac{p \Psi_{2}}{R_{2}} \\
& \Psi_{2}=L_{m} i_{m 1}+L_{r}\left(\frac{-p \Psi_{2}}{R_{2}}\right) .
\end{aligned}
$$


Thus, there is

$$
\Psi_{2}=\frac{L_{m}}{1+T_{2} p} i_{m 1}
$$

where $T_{2}=L_{r} / R_{2}$ denotes time-constant of rotor excitation, as we can see, rotor flux $\Psi_{2}$ is only produced by $i_{m 1}$ and has nothing to do with $i_{t 1}$, therefore, $i_{m 1}$ is known as the component of the stator excitation.

Similarly, there is

$$
\omega_{s}=\frac{L_{m}}{\psi_{2} T_{2}} i_{t 1}
$$
(2.11) is

Regarding the equation of torque, the solution of simultaneous formula (2.10) and

$$
T_{e}=n_{p} \frac{L_{m}}{L_{r}} \Psi_{2} i_{t 1}
$$

From formula (2.16) we can see that because rotor flux $\Psi_{2}$ is only produced by $i_{m 1}$ and has nothing to do with $i_{t 1}$, when $\Psi_{2}$ is permanent, torque $T_{e}$ only has something to do with the component of torque of stator current $i_{t 1}$, amounting to the armature current of DC motor, we achieve a good solution of decoupling.

How to get the direction of rotor flux linkage vector $\Psi_{2}$ is one of the technical difficulties of vector control algorithm of asynchronous motor. This paper introduces current hall sensor to measure the instantaneous value of motor's three-phrase stator current $i_{A}, i_{B}$, $i_{C}$ and introduces photoelectric encoder to measure the signal of rotational speed $\omega$. Then according to the coordinate conversion of formulas (2.1) and (2.2) and the equation of motor, design the magnetic flux viewer, through the indirect method calculate to obtain the size of $\Psi_{2}$ and the direction of $\phi$.

According to the decoupling mathematical model of induction motor, make use of Matlab/Simulink to build simulation model of vector control system of slip type of induction motor that mainly the contain following modules.

(1) The module of rotational speed controller: achieve closed-loop control of motor rotational speed, introduce traditional PI controller, the module's input is rotational speed demand signal $\omega^{*}$ and rotational speed return signal $\omega$. Take the error between $\omega^{*}$ and $\omega$ as the input of PI controller, through the set proportion parameter $K_{P}$, integral parameter $K_{I}$ and torque amplitude limiter calculate the torque given value $T_{e}^{*}$.

(2) The calculated module through the component of stator current magnetic flux and torque: based on vector control system of rotor flux-oriented design, so flux linkage introduces open-loop control, in the steady state, there is

$$
i_{s M}^{*}=\frac{\Psi_{r}^{*}}{L_{m d}} .
$$




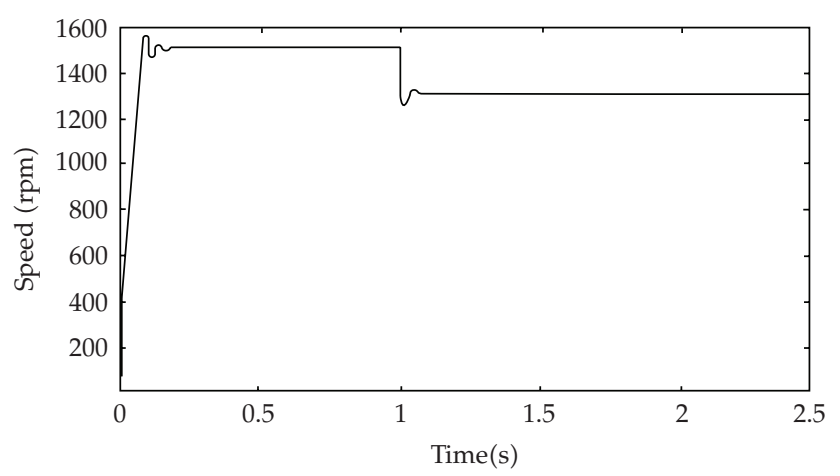

Figure 2: The simulation waveform of motor no-load startup on the first second adding load.

Thereby, according to the set flux linkage $\Psi_{r}^{*}$ calculate the magnetic flux component of stator current. Then, according to the given torque of the module of rotational speed controller and the given flux linkage, by the equation of torque control of formula (2.16) calculate the torque direction of stator current.

(3) The control module of current hysteresis loop: draw a comparison between the given value of stator three-phrase current and actual value and obtain a deviation, the deviation passes the high-gain amplifier with hysteresis loop character, that is, the hysteresis loop comparator DHC, and compares with the set maximum current deviation to control the make-and-break of two of the upper and lower bridge arm of each phrase of inverter. Make the actual current continually track the waveform of the given current and fluctuate only in the range of deviation.

In addition, the simulation structure also includes the calculation module of the magnetic field orientation angle, the module of $2 / 3$ converter, the module of inverter, the module of induction motor, and so on. Figure 2 is no-load startup and waveform of rotor palstance when time is the first second adding $50 \mathrm{~N} \cdot \mathrm{m}$ load. We can see when the motor load changes the speed responses very quickly, and the system has good dynamic following and reaches the stable state quickly, which proves the feasibility of oriented vector control based on rotor flux linkage.

\section{Controller Design}

\subsection{Controller Hardware Design}

Based on the technical requirements of Hybrid Electric Vehicle motor controller, introduce the special purpose chip of motor control of Microchip Corporation's, dspic33FJ128MC706, to be the controller core and according to the pulse width modulation signal of voltage of asynchronous induction motor generated by the manner of space vector modulation carry on the overall design of the controller. Asynchronous induction motor parameters: rated power $P_{N}=55 \mathrm{kw}$, rated current $I_{N}=300 A$, rated speed $n_{N}=2500 \mathrm{rpm}$, rated torque $T_{e}=200 \mathrm{~N} \cdot \mathrm{m}$. following.

The hardware structure of motor controller is shown in Figure 3, mainly including the 


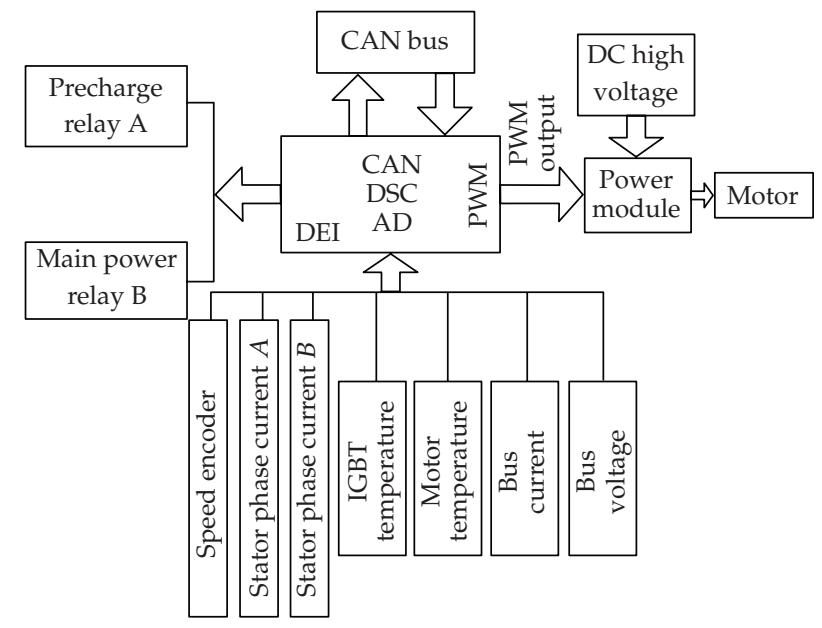

Figure 3: The block diagram of motor controller hardware.

(1) Main circuit: the main circuit of controller introduces intelligent power module (IPM) of IGBT to constitute inverter circuit of three-phrase full-bridge and combines of control algorithms for motor control to achieve the following basic functions.

(i) Load matching: the controller's output power matches the selected motor, not only meet the requirements of electrical rating values, but also ensure that the motor can run in short-term overload.

(ii) Four-quadrant operation: when Hybrid Electric Vehicle is in the run-time, the forward and reverse drive motor as well as the back coupling of the regeneration energy is the normal running status, requiring that the motor can operate in four-quadrant.

(iii) High reliability and stability.

(2) Power supply module: provide $15 \mathrm{~V}, 5 \mathrm{~V}, 3.3 \mathrm{~V}$ power supply for controller and its peripheral devices and complete the power supply monitoring function.

(3) The A/D module: it is responsible to convert multichannel analog quantity into digital quantity, such as the temperature of motor stator, the radiator temperature, the current of motor stator, DC bus, and voltage, which is provided for DSC to calculate and real-time monitor the state of controller and motor as well as providing the necessary parameters $i_{A}$ and $i_{B}$ for vector control. The isolation of analog signal uses the precise capacitive isolation amplifier ISO124.

(4) The module of input-output isolation: use optoelectronic isolator TLP521-4/2 to isolate with DSC.

(5) The DEI module: process the signal from the speed sensor and calculate the motor speed, providing the necessary parameter $\omega$ for vector control.

(6) The PWM module: export the PWM signal through optocoupler isolation to IGBT drive module, and control the breakover of IGBT's up and down bridge arms to achieve the control of motor. 
(7) The module of CAN controller: define a node of CAN bus on the network to achieve the communication among motor controller, power train controller, and battery management system. In order to increase the communication distance, improve the system's instantaneous anti-interference ability, protect bus, reduce radio frequency interference (RFI), and achieve thermal protection; this paper increases CAN transceiver chip PCA82C250 between the module of CAN controller and CAN bus. Considering the car's bad environments, to further enhance the antiinterference measures, between the two CAN notes also adds to the isolation circuit being consisted of high-speed optocoupler 6 N137.

(8) Protection circuit: the controller controls the motor's operation, needing real-time monitor motor and its state, including the temperature of the motor stator, the temperature of the inverter radiator, and DC busbar voltage and current. After being converted by the A/D the analog signals compare with the comparator, when there is abnormal situation, for example, the motor load is too high, the back coupling of the energy is too high and illegal operation when debugging, the protection signals after reversed-phrase enter the breakdown input pin of DSC. The software turns off IGBT and coordinates with the IPM's hardware protection to achieve controller's protection function.

(9) The electromagnetic interference suppression: on the basis of analyzing a variety of electromagnetic interference of HEV, introduce different means to enhance the anti-interference ability of electromagnetic sensitive devices such as CAN bus; the detail will be shown in Section 4.

(10) Design for controller vibration isolation: in order to reduce the effects of vibration by the frame to controller, according to GB HG/T3080-1988 (the shockproof rubber material uses rubber material), select the D11-type rubber which has vibration attenuation property to design rubber vibration isolator, and carry on the multidegree-of-freedom vibration model checking to ensure that in the motivation of a simulation road the amplitude and acceleration of controller meet the requirements of vibration.

\subsection{Design for Controller Software}

Controller software mainly uses $\mathrm{C}$ language and assembly language to develop under the MPLABC30 environment, of which the main program, interrupt handling, CAN communication program use $C$ language, because $C$ language makes the whole process frame structure clear, easy debugging, and maintenance, in line with the concept of structural process design. The related core algorithm like vector control algorithm completely uses the assembly language, which can optimize the code structure, significantly reduce the process running time, improve operating efficiency, and ensure real-time control.

In order to make the software architecture clear, easy debugging, and maintenance, introduce modular design concept, according to the function divide into the module of the main program, vector control, CAN communication, A/D sampling, I/O input-output. The flow of the main function is shown in Figure 4. The controller is electrified to carry out the main function. Initialize clock, peripheral, interface and be connect to $24 \mathrm{~V}$ power supply, precharge power capacitors as well as the main power supply relay turns on, then enter the major cycle. Monitor IGBT radiator, the motor temperature, DC bus bar current, and voltage 


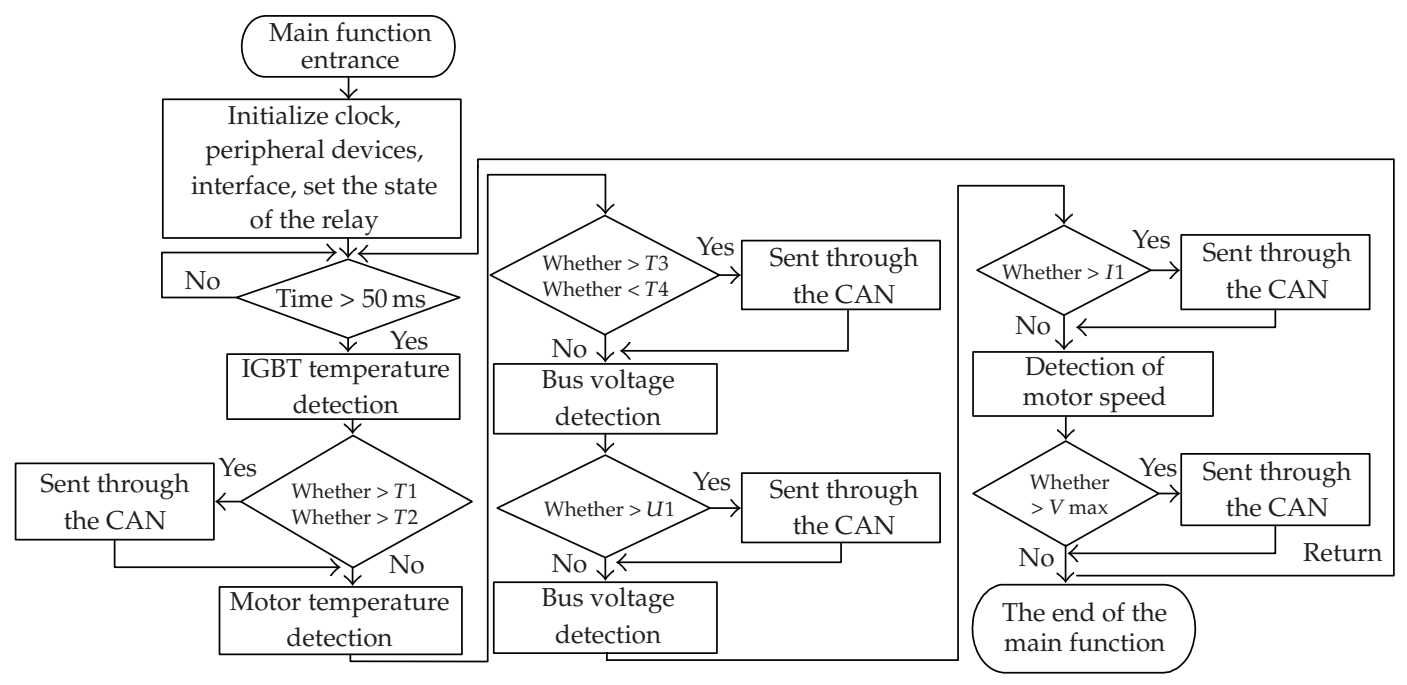

Figure 4: The main function flow chart.

value. At the same time, momentarily respond the interrupt, complete the update of vector control parameters and the communication of CAN with power train controller.

\section{Research of Motor Controller's Electromagnetic Compatibility}

Compared with the traditional vehicle, HEV has the following characteristics in the control aspect. The control objects are more decentralized, data exchange between the various units is more frequent, and the requirements of reliability and real-time of data exchange are higher. Hence, it is necessary to introduce the simple, efficient, and reliable communication in the HEV.

CAN bus, a serial bus, have the merits of simple structure, fault-tolerant capability, technical maturity, and so forth, which is widely used in the field of automotive electronic control. HEV needs more equipments and higher coordination control, therefore, the use of CAN bus achieving in-vehicle network system is a good solution. All of the Prius of Toyota, the Precept of GM, and the Prodigy of Ford use the CAN bus.

The HEV drive motor of this project is AC asynchronous motor, being driven by the inverter. The power module, (insulated gate bipolar transistor) IGBT, is widely used in the inverter, which is a dual current-carrying device and whose switching characteristics are controlled by the gain of PNP transistor. Therefore, in essence, the current peak of IGBT will be higher than that of (metal-oxide semiconductor field effect transistor) MOSFET. Meanwhile, the carrier frequency of the inverter is up to $20 \mathrm{kHz}$ and bus bar voltage is over a hundred volts, so when there is (pulse-width modulation) PWM wave, there will be a very high voltage peak value, which will inevitably lead to serious interference noise of conduction and electromagnetic radiation [9-11]. In addition, the number of automotive electronic equipment increases day by day, which make the electromagnetic environment of the HEV complex and poor. So, it is of great significance to analyze the system's electromagnetic interference source and its propagation path, take the corresponding measures to make electromagnetic sensitive devices, such as air bag and CAN bus network, work regularly 


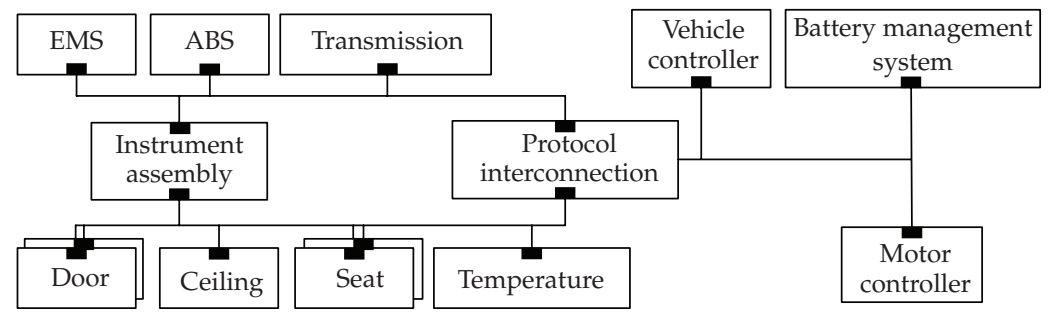

Figure 5: Frame of HEV CAN bus.

under strong interference condition. The experimental results indicate that the antijamming measures are practical and the system operates well.

\subsection{Hybrid Electric Vehicle CAN Bus System}

The in-vehicle network system of HEV of this project is composed of two major parts, which are connected by the protocol interconnection component and consist of unitive CAN bus network. The basic structure is shown in Figure 5.

The first part includes three node controllers, that is, motor controller, vehicle controller, and battery management system. Each node controller can independently accomplish the function of command decomposition and transfer bottom layer instruction. In this way, for an action unit, the node controller will act as "command host."

The second part includes body measurement and control points, such as car door, ceiling, seat, and the temperature control point, meanwhile include the communication among engine, ABS, and transmission and transfer the data to automobile instrument assembly.

The protocol interconnection component: this part is responsible for the connection of the above two parts. Although these two major parts use the CAN bus network and their physical layer and partial data link layer are established on the CAN2.0B, partial data link layer and application layer protocol due to controlled device use different communication protocols. Therefore, introduce the protocol interconnection component to connect the bus protocol of the two segments so as to consist of unitive bus control network.

Obviously, the CAN bus network system is very important, in relation to vehicle safe information, such as EMS, ABS, and other related information, must be timely, reliable transmission. Due to covering the entire body, its performance and reliability directly involve the normal work of the entire automotive.

\subsection{Analysis of System Electromagnetic Interference}

Analysis of the source of electromagnetic interference and its propagation path is very important for solving the problem of system electromagnetic compatibility. Use the asynchronous motor, power up to $30 \mathrm{KW}$, for debugging experiments. At first, carry on the point-to-point communication debugging, the power supply voltage of bus bar is $48 \mathrm{~V}$. After using the conventional antijamming measures, CAN bus system can be normal communication. However, when we do the bench experiment, the power supply is $336 \mathrm{~V}$ battery pack, there will always present the phenomena of CAN bus inaccessibility and drop 


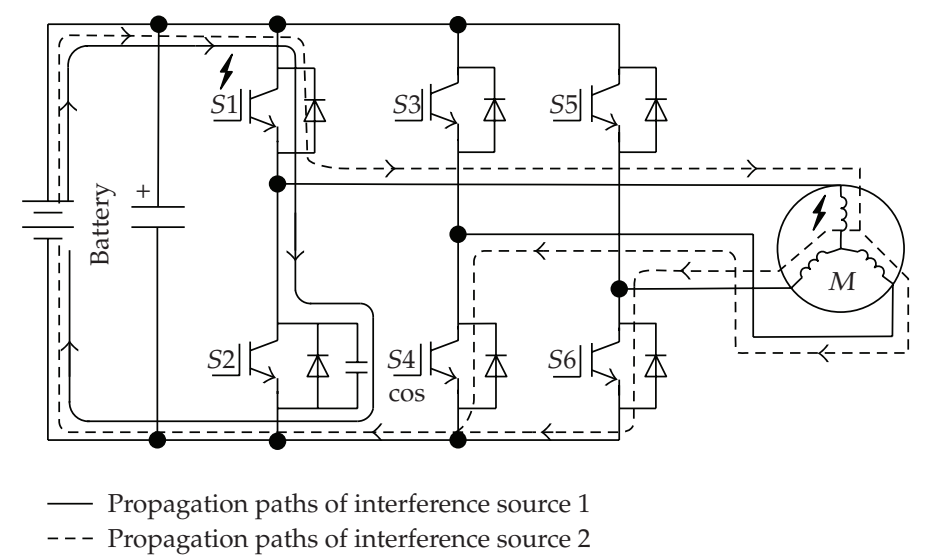

Figure 6: Differential mode interference sources and their propagation paths.

frames during data transmission. This indicates that when the inverter operates under the rated conditions, there will be a very strong electromagnetic interference which seriously affects the normal communication of the CAN bus system by conduction and radiation. The experiment shows that the main sources of electromagnetic interference include the following aspects.

\section{(1) Differential Mode Interference}

Make the bus bar as the interference of shuttle route, which forms the loop through bus bar, battery, and inverter. By the high frequency equivalent circuit of the three-phrase inverter, when IGBT switch acts form a kind of differential mode interference source [12], which returns to battery through bus bar. Besides, when the motor is high frequency voltage, the stator coil will produce voltage peak, which forms another differential mode voltage through bus bar [13]. Both measure battery terminal voltage and motor phase voltage and carry on Fourier transformation and compare or use the linear impedance stabilization network series in the bus bar can analyze the two differential mode interferences. Its propagation path is shown in Figure 6.

\section{(2) Common Mode Interference}

The interference forms through the parasitic capacitance of phase and GND and forms the loop again through GND. When the IGBT switch acts, there will be not only differential mode interference voltage but also common mode interference voltage [12]. The common mode interference voltage, through the parasitic capacitance of IGBT base relative to the radiator, forms the common mode interference current flowing to the chassis. Besides, form the voltage peak in the stator coil, at the same time induce the shaft bearing voltage, which produces the shaft bearing current flowing to the chassis through the motor shaft, thus form another common mode interference current [13]. Measure the current and carry on Fourier transformation, obtain its propagation path as shown in Figure 7. 


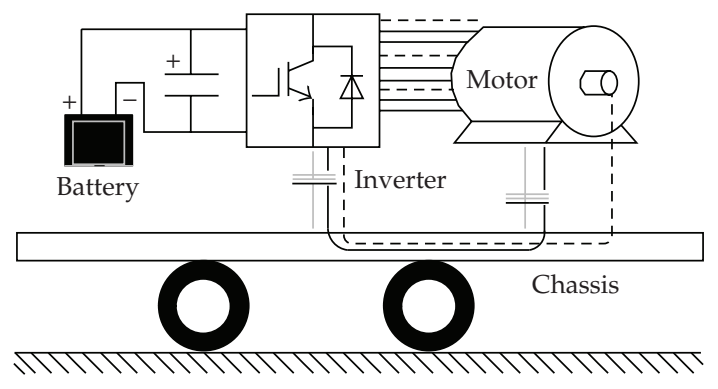

_ Propagation paths of interference source 1

Figure 7: Common mode interference sources and their propagation paths.

\section{(3) radiated Interference}

The interference source takes the energy to radiate to the surrounding space in the form of electromagnetic wave. The experiment shows that around the inverter and the entire chassis the radiation intensity is very high, even when two vehicles approach, the electromagnetic radiation level will intensify. Because the differential mode interference formed in the inverter radiate energy to the surrounding space, and because the common mode current flowing to the chassis forms the current loop on its surface due to the conductor skin effect, there will be very strong electromagnetic interference. Obviously, the best way to suppress the radiation interference is to weaken the intensity of differential mode and common mode interference.

In addition, when the generator commutation or load current change suddenly, there will be electromagnetic wave. The switching frequency, the multiples of switching frequency, and high frequency harmonic of the PWM signal will produce radiation interference.

\subsection{Solutions for the Problem of System Electromagnetic Interference}

Practice shows that the occurrence of any electromagnetic interference must have three elements of interference source, propagation path, and sensitive devices. Any element is weakened or lost, the problem of electromagnetic interference will be improved or solved. Based on the above analysis, we take the following antijamming measures.

\section{(1) Attenuation of Differential Mode Interference and Radiation Interference}

Introduce the multilayer printed power circuit board to suppress the differential mode interference in the inverter and the corresponding electromagnetic radiation. The results of the experiment indicate that the frequency of the differential mode interference is $10-20 \mathrm{M}$. By formula (4.1), $\delta$, the skin depth of interference on copper, is 0.01-0.02 mm. All of the interference due to conductor skin effect flow from the conductor surface

$$
\delta=\sqrt{\frac{1}{\pi f \mu \sigma}},
$$


where $f$ denotes the interference frequency, $\mu$ denotes the permeability of copper, $\mu=4 \pi \times$ $10^{-7} \mathrm{H} / \mathrm{m}, \sigma$ denotes the electrical conductivity of copper, $\sigma=5.8 \times 10^{7} \mathrm{~S} / \mathrm{m}$.

Thus, the effect of traditional filter with lumped parameter circuit model is not good. The multilayer power circuit board is a sandwich structure. The second and third layers are used to take the bus bar current, the symmetrical structure, appropriate wire width, and dielectric substance thickness form the reasonable coupling capacitance, which is used to absorb the differential mode interference entering the bus bar to reduce the corresponding radiation energy.

\section{(2) Attenuation of Common Mode Interference and Radiation Interference}

Introduce the appropriate ground impedance to attenuate the common mode interference current flowing to the ground and the corresponding electromagnetic radiation. Usually, only the tires of the vehicle contact with the ground, so the whole body is insulated to the ground. Being known as the previous analysis, the common mode interference current flows to the chassis through the inverter radiator base or motor shaft, its voltage is likely to endanger the safety of the passengers. Therefore, the radiator base and motor enclosure must be grounded. The grounding impedance must be appropriate to furthest attenuate the common mode interference current. The value of $R, C, L$ can be calculated by the following two formulas:

$$
\begin{aligned}
& i^{\prime}=\frac{E}{\sqrt{L / C}} \varepsilon^{-1 / T^{t}}, \\
& L=\sqrt{\frac{\sqrt{L / C^{2}}}{4 \pi^{2} f_{c}^{2}+(R / 2 L)^{2}}},
\end{aligned}
$$

where $i^{\prime}$ denotes the peak common mode interference current flowing to the chassis, $E$ denotes common mode voltage, $T$ denotes the time constant of peak current, the size is $R / 2 L$, $f_{c}$ denotes the frequency of peak current $i^{\prime}$.

Measure the common mode interference current flowing to the chassis and carry on Fourier transformation to find out peak current $i^{\prime}$, its amplitude is $E / \sqrt{L / C}$. $E$ is common mode voltage, which usually takes one-third of bus bar voltage, thus obtain $\sqrt{L / C} \cdot(R / 2 L)$ can be estimated through time constant of peak current, calculate $L$ by formula (4.3), then figure out $R, C$. Meanwhile, consider the upper limit of safe voltage. The experiment indicates that the increase of grounding impedance is helpful to reduce common mode current, but simultaneously enhance the potential of motor enclosure.

\section{(3) $\pi$-Type Filter Circuit Improves the CAN Antijamming Ability}

This filter circuit ingeniously uses the parallel resonant characteristic of inductance coil and capacitance, appropriate to adjust its value to make frequency characteristic curve steep and peak high. Because the coil impedance is very small, the signal waveform will not distort when suppress the noise. Insert loss to indicate the filter's attenuation to the interference, use 
$I L$ to express. It is related to the parameters of inductance, capacitance, noise frequency and so on, the relationship is shown as follows:

$$
I L=20 \lg \left[\frac{\omega^{2} L C+\left(L C^{2} \omega^{3}+2 \omega C\right) R_{L} R_{S}}{R_{S}+R_{L}}\right],
$$

where $R_{L}$ denotes load impedance, $R_{S}$ denotes source impedance, $\omega$ denotes noise angular frequency, $L$ denotes inductance value of coil, $C$ denotes the size of capacitance. In view of the corresponding interference frequency and the requirements of $I L$, calculate the parameter value of $\pi$-type filter. Considering the inaccurate estimates of load impedance $R_{L}$ and source impedance $R_{S}$ and the impact of parasitic capacitance on inductance coil when it is high frequency, the calculated value needs to adjust according to the actual debugging effect.

\section{(4) The Matching Resistance Improves the CAN Antijamming Ability}

Appropriate choice of matching resistance can greatly improve the antijamming ability of CAN bus system. The signal at the end of the transmission line suddenly encounters very small cable impedance, then it will cause reflection in this place and create the waveform distortion. In theory, as long as connecting the terminal resistance matching cable characteristic impedance at the end of the transmission cable, there will not present the phenomenon of signal reflection. However, in fact, the terminal resistance of the whole communication system is also affected by the number of node, the impedance of each node, capacitance and bus capacitance, and it is often different from the recommendation value $120 \Omega$ of the twisted-pair terminal matching impedance. When debugging let the sending end send data $55 \mathrm{H}$ in use of the actual baud rate, at the receiving end use the oscilloscope to observe the output square wave, adjust the matching impedance to obtain the best waveform whose amplitude voltage is no less than $200 \mathrm{mV}$, this value is the best matching impedance.

\section{Experimental Results}

\subsection{CAN Communication Debugging}

In the HEV complex electromagnetic environment, the real-time, reliability, and accuracy of the CAN communication must be first assured. In the third part of the electromagnetic compatibility of motor controller, analyze the source of electromagnetic interference and its propagation path in detail. In order to verify whether the used antijamming measures are reliable and effective, the following experiments have been done.

\section{(1) The Waveform Debugging of CAN Bus Communication}

To have a direct-viewing understanding of the antijamming effect of CAN bus communication, use digital oscilloscope to observe the communication waveform. As shown in Figure 8, after adding the antijamming measures, the waveform quality is improved obviously. 


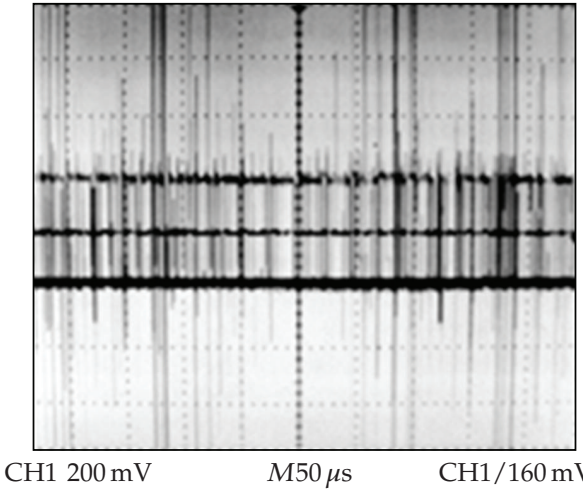

(a)

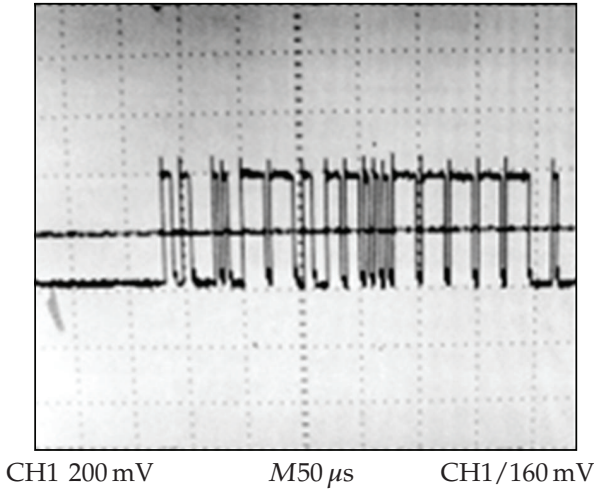

(b)

Figure 8: The CAN wave before and after taking antijamming measures.

Table 1: The experiment results of error rate.

\begin{tabular}{lccc}
\hline & Speed $/(\mathrm{kbps})$ & Transmission distance $/(\mathrm{m})$ & Bit error rate \\
\hline Take no measures & 128 & 20 & $<10^{-6}$ \\
Take no measures & 512 & 20 & $<10^{-4}$ \\
Take measures & 128 & 20 & $<10^{-8}$ \\
Take measures & 512 & 20 & $<10^{-6}$ \\
\hline
\end{tabular}

\section{(2) The Experiment of Error Rate of CAN Bus Communication}

In order to verify the stability of CAN bus system after using the above antijamming measures, design and carry on the following experiment. Take two nodes from the bus to carry on the data transmission experiment, the nodes connect with the upper monitor through CAN and USB converter interface. The node 1 sends quantitative bits data to the node 2 in accordance with predetermined data format. On the node 2 the software of upper monitor compares the transmitter and receive data and analyzes the wrong bits. Table 1 is the experiment result. After using the corresponding antijamming measures, the error rate of the communication decreases two orders of magnitude.

\subsection{The Experiment of the Control Logic}

During the process of controller controlling motor, in order to assure the safety of the system, the control logic, such as power-on sequence and fault treatment, is particularly important. The power-on sequence is the controller of power train sends self-checking instruction to each controller, when the battery management system receives the self-checking instruction, it will carry on self-checking, then connect the relays of all of the battery packs, the battery packs precharge the capacitance through current-limiting resistance; the motor controller is poweron, self-checking, after completion sends the ready instruction to the controller of power train, the controller of power train sends the enabled instruction, when the motor controller receive the enabled instruction, connect the capacitance precharge relay and short current-limiting resistance, and connect the main relay to electrify the controller, at this point the controller will be able to start the motor running. The logic of fault treatment is that controller realtime 


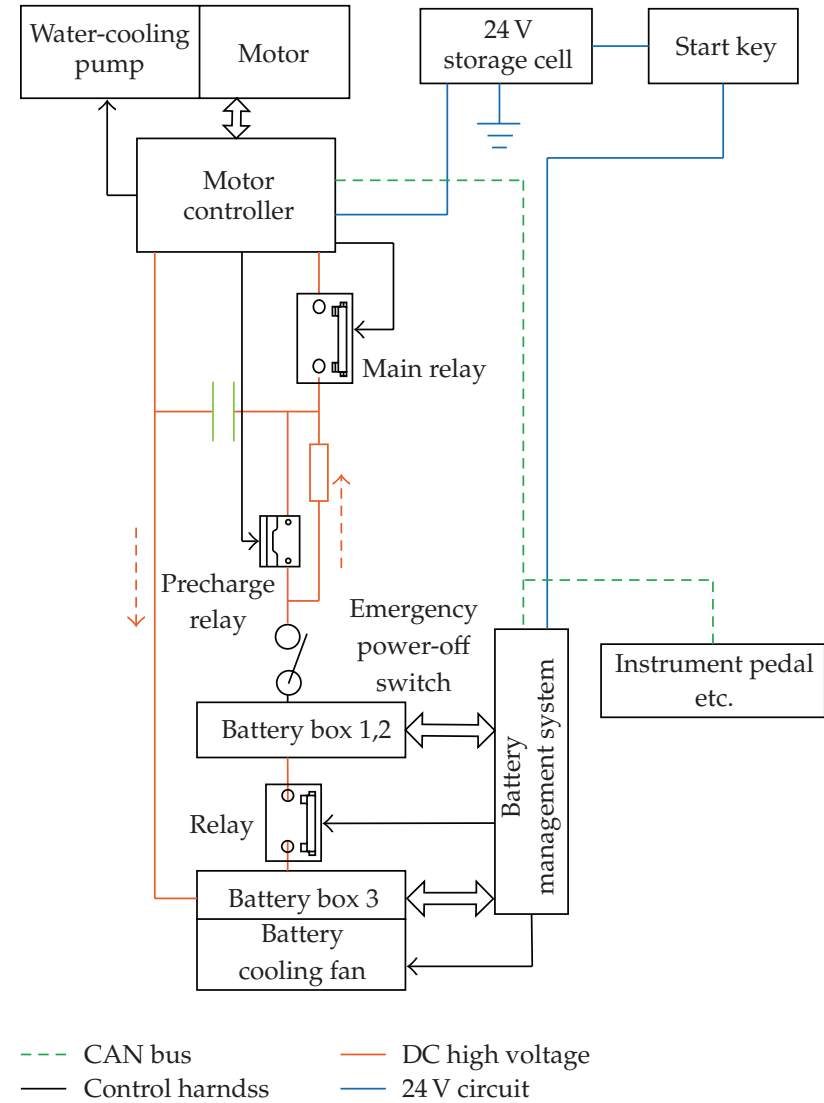

Figure 9: The principle diagram of test-bench.

monitors the voltage and current of DC bus bar, the temperature of rotor and controller, when overruns, immediately cut the output of PWM and close IGBT and the main relay to ensure the safety of the whole system.

\subsection{The Motor Bench Experiment}

The motor drive system of HEV need has the performance of low speed and high torque, a wide range of variable speed, strong overload capacity, fast torque output respond, high efficiency of multipoint operating mode, and so on. Obviously, the performance of motor and controller and their matching are very important. Therefore, after completing the hardware debugging of motor controller and the main software writing, carry on the bench experiment by controller driving motor.

The bench experiment can effectively detect whether the performance of the motor and its controller meet the design requirements, and it is the most effective measure to assure motor and its controller work safe and reliable in the vehicle and to reduce the unnecessary trouble of debugging on board. The bench of this paper is constituted of motor, controller, cooling system, electric eddy current dynamometer, upper monitor, power battery pack, and its management system, as shown in Figure 9. The dynamometer is connected with motor, as 
Table 2: The motor performance parameters.

\begin{tabular}{lcc}
\hline Type & \multicolumn{3}{c}{ YPQ160M-4 (55kW) } \\
\hline Performance parameters & Design value & Trial value \\
Peak power (kW) & 110 & 110 \\
Peak power efficiency (\%) & 89 & 89.2 \\
Peak power slippage (\%) & 4.5 & 4.45 \\
Overload power (kW) & 83 & 83 \\
Overload power efficiency (\%) & 91 & 91.15 \\
Overload power slippage (\%) & 3.5 & 3.4 \\
Rated power (kW) & 55 & 55 \\
Rated power efficiency (\%) & 92.5 & 92.8 \\
Rated power slippage (\%) & 2.5 & 2.43 \\
Half-power (kW) & 27.5 & 27.5 \\
Half-power efficiency (\%) & 92.4 & 92.55 \\
Half-power slippage (\%) & 1.5 & 1.4 \\
\hline
\end{tabular}

upper monitor software can realize the real-time measurement and record of motor torque and rotational speed. The controller controls the motor operation and communicates with power train controller with CAN to get the request torque information, at the same time manages the motor and controller's cooling system. Power battery pack is divided into three groups and are in series by relays, the battery management system monitors and manages its state.

When reverse the key to electrify, the battery management system connects the battery's internal relay, and the battery precharges the capacitor through current-limiting resistance; then controller makes precharge relay connect, shorts charging resistance and connects main relay to electrify the controller, at this time controller can start motor running. In order to prevent accidents from happening, during the experiment add an emergency power-off switch, which can be artificially cut off the battery power supply, to fully guarantee the security of the entire system.

According to "Electric vehicle motor and its controller Part 2: test methods" (GB/T 18488.2-2006), carry on the experiment of torque-speed characteristic, power, efficiency, slip. The experimental data is shown in Table 2, the torque-speed curve is shown in Figure 10.

Note. In Figure 9, in order to conveniently illustrate, draw capacitor, main relay, precharge capacitor, and current-limiting resistance outside of controller which originally locate in controller.

On the analysis of the experimental data and motor characteristic curve, the performance parameters of the motor rated power, overload power, peak power and slip can reach or approach the design requirements, and the system efficiency of the selected experimental points are all above $89.2 \%$; that is, the comprehensive efficiency is high, this is very important for HEV sometimes light load, sometimes heavy load and sometimes overload. On the condition of overload motor can constantly output at torque $T=300 \mathrm{~N} \cdot \mathrm{m}$ and speed $n=0-2500 \mathrm{rpm}$, on the condition of rated torque $T_{e}=200 \mathrm{~N} \cdot m$ motor can even remain the speed $n=3500 \mathrm{rpm}$, which is higher than the rated speed $n_{e}=2500 \mathrm{rpm}$. What we can see is that the drive system not only can output high torque $T=300 \mathrm{~N} \cdot \mathrm{m}$ in low speed, but also can govern speed in a wide range $n=0-3500 \mathrm{rpm}$ in the rated torque. 


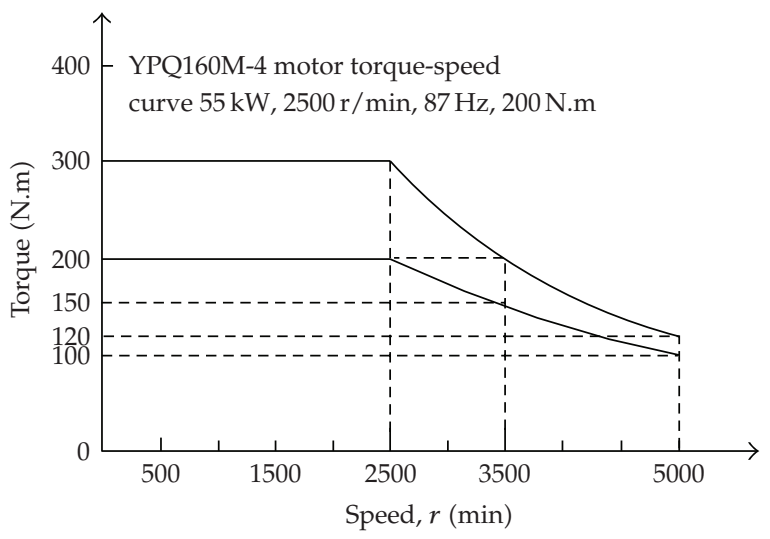

Figure 10: Motor torque-speed characteristic curve.

\section{Conclusion}

Nowadays, research of Hybrid Electric Vehicle is one of the most meaningful ways to solve the problems of pollution and energy. Motor and its control technology are one of main components of Hybrid Electric Vehicle, and largely affect vehicle's power performance, fuel economy, and emission. This paper carries on the research of motor control technology of application HEV, designs HEV motor controller based on vector frequency conversion technology, and completes the controller's software and hardware design. In accordance with the characteristics of the HEV electromagnetic interference source, propagation path, and CAN bus communication, this paper proposes the corresponding antijamming measures, which greatly improves the antijamming ability of the CAN bus system and controller working stability. Finally, the motor bench experiment verifies that the designed driving system has the performance of low speed and high torque, a wide range of variable speed and high comprehensive efficiency, which create advantages for system dynamic performance experiment, durability experiment and vehicle road experiment.

\section{Acknowledgment}

This work is supported by the 863 National High Technology Research and Development Program of China under Grant 2008AA11A140.

\section{References}

[1] Q. Q. Chen, F. C. Sun, and J. G. Zhu, Modern EV Technology, Beijing Institute of Technology Press, Beijing, China, 2002.

[2] H. Hu and H. Song, Electric Vehicle, China Communications Press, Beijing, China, 2003.

[3] R. Saeks, C. J. Cox, J. Neidhoefer, P. R. Mays, and J. J. Murray, “Adaptive control of a hybrid electric vehicle," IEEE Transactions on Intelligent Transportation Systems, vol. 3, no. 4, pp. 213-233, 2002.

[4] W. Liu, G. W. Cheng, and C. N. Deng, "Analysis of electric vehicle's driving motor," Motor Technology, pp. 26-28, 2006.

[5] H. D. Li, The Control System of AC Speed-Governing, Publishing House of Electronics Industry, 2003.

[6] K. Zhou and D. Wang, "Relationship between space-vector modulation and three-phase carrier-based PWM: a comprehensive analysis," IEEE Transactions on Industrial Electronics, vol. 49, no. 1, pp. 186-196, 2002. 
[7] S. Shinnaka, S. Takeuchi, A. Kitajima, F. Eguchi, and H. Haruki, "Frequency-hybrid vector control for sensorless induction motor and its application to electric vehicle drive," in Proceedings of the IEEE Applied Power Electronics Conference and Exposition (APEC'01), vol. 1, pp. 32-39, 2001.

[8] Z. X. Fu, J. Xiang, W. C. Reynolds, and B. Nefcy, "Vector control of an IPM synchronous machine capable of full range operations for hybrid electric vehicle application," in Proceedings of the IEEE Industry Applications Society Conference (IAS '03), vol. 3, pp. 1443-1450, 2003.

[9] D. A. Rendusara and P. N. Enjeti, "An improved inverter output filter configuration reduces common and differential modes $\mathrm{dv} / \mathrm{dt}$ at the motor terminals in PWM drive systems," IEEE Transactions on Power Electronics, vol. 13, no. 6, pp. 1135-1143, 1998.

[10] N. Mutoh, J. Nakashima, and M. Kanesaki, "Multilayer power printed structures suitable for controlling EMI noises generated in power converters," IEEE Transactions on Industrial Electronics, vol. 50, no. 6, pp. 1085-1094, 2003.

[11] Y. Onozawa, M. Otsuki, and Y. Seki, "Investigation of carrier streaming effect for the low spike fast IGBT turn-off," in Proceedings of the 18th International Symposium on Power Semiconductor Devices E IC's, pp. 173-176, University of Naples, Naples, Italy, 2006.

[12] C. C. Chen and X. Y. Xu, "Modeling the conducted EMI emission of an electric vehicle (EV) traction drive," in Proceedings of IEEE International Symposium on Electromagnetic Compatibility, vol. 2, pp. 796 801, Denver, Colo, USA, 1998.

[13] N. Mutoh, M. Nakanishi, M. Kanesaki, and J. Nakashima, "Control methods for EMI noises appearing in electric vehicle drive systems," in Proceedings of the IEEE Applied Power Electronics Conference and Exposition (APEC '05), vol. 2, pp. 1022-1028, Austin, Tex, USA, 2005. 


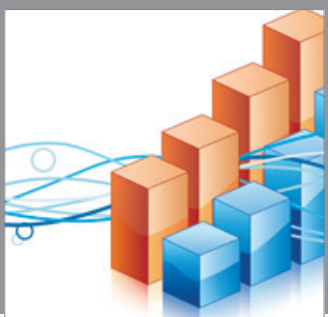

Advances in

Operations Research

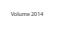

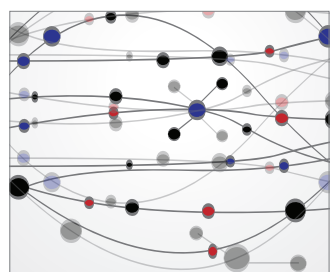

\section{The Scientific} World Journal
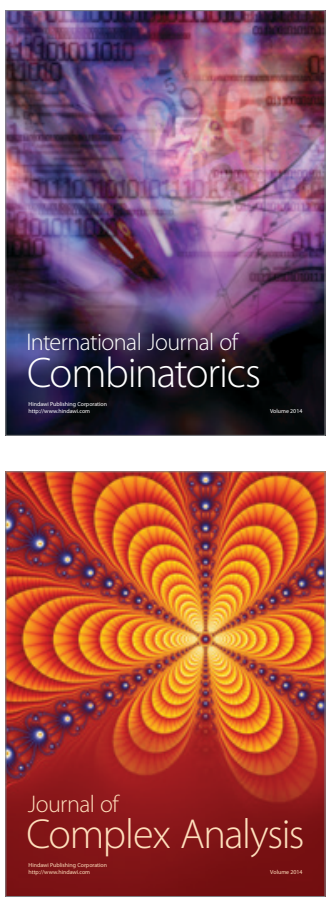

International Journal of

Mathematics and

Mathematical

Sciences
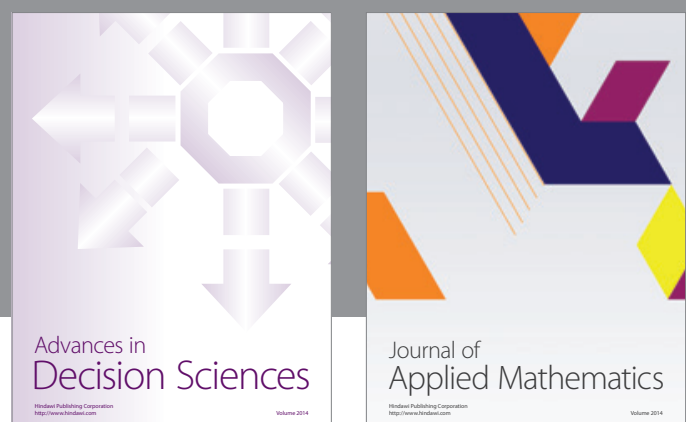

Journal of

Applied Mathematics
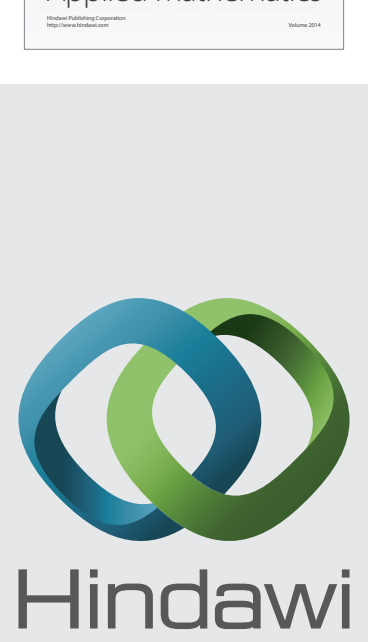

Submit your manuscripts at http://www.hindawi.com
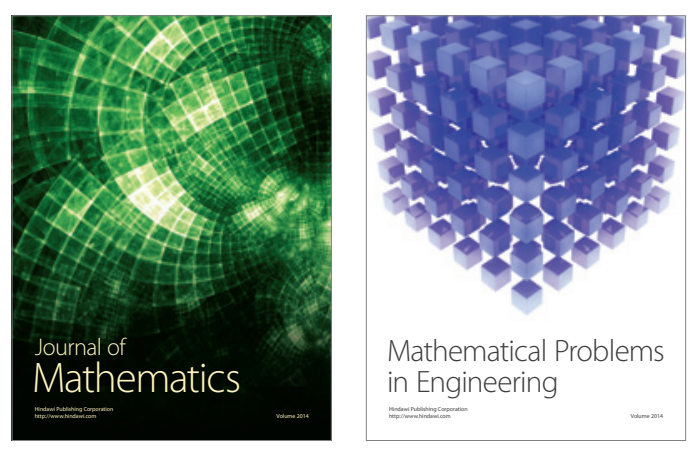

Mathematical Problems in Engineering
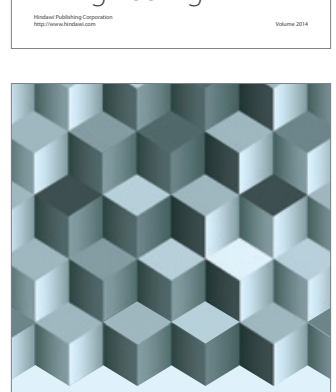

Journal of

Function Spaces
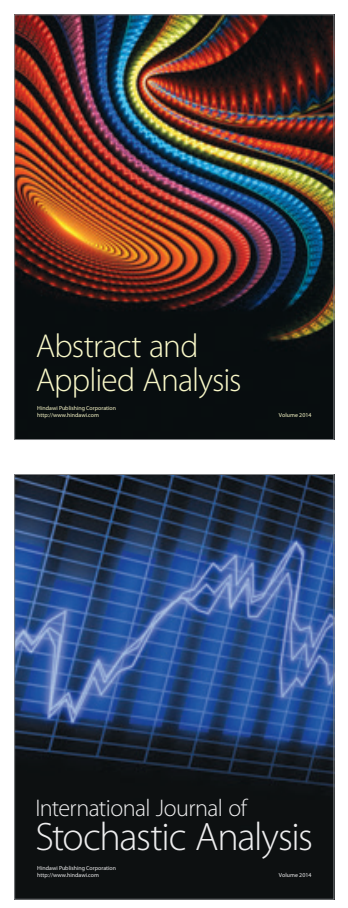

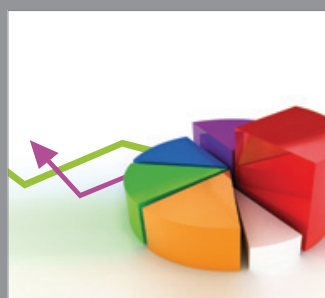

ournal of

Probability and Statistics

Promensencen
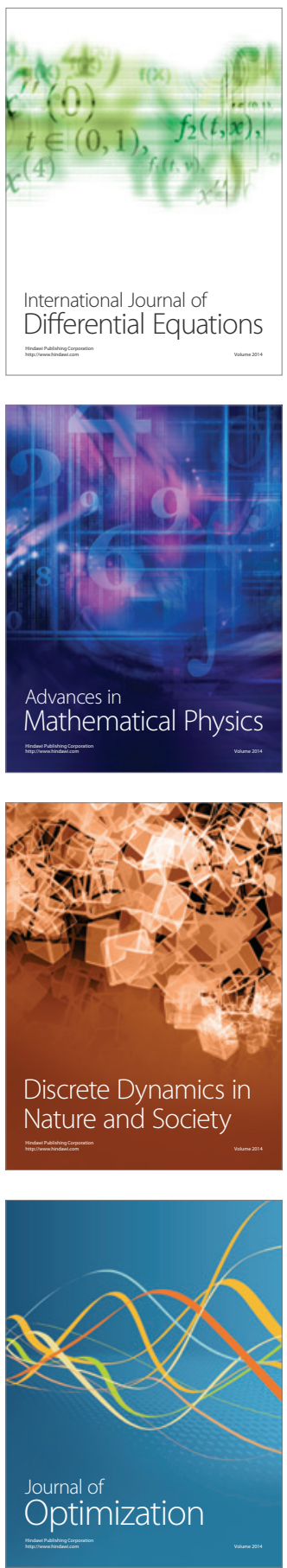